\title{
Colonic tuberculosis masquerading as colon cancer
}

Authors: A Kumar, M Patodia, PK Pandove, VK Sharda

Location: Rajindra Hospital, Patiala, Punjab, India

Citation: Kumar A, Patodia M, Pandove P K, Sharda V K. Colonic tuberculosis masquerading as colon cancer. JSCR 2012. 5:10

\section{ABSTRACT}

Isolated colonic tuberculosis (TB) is rare, and the symptoms are nonspecific making early diagnosis and management difficult. Although colonoscopy and biopsy is an important diagnostic modality, the features are variable and the distinction from other conditions of the colon, especially Crohn's disease and cancer, may be impossible without surgical resection. We report a case of ascending colon TB which was mistaken for colonic cancer during colonoscopy, with non-specific results on biopsy. The diagnosis was finally made when the histopathology report was received. The diagnostic dilemma of colonic TB is discussed.

\section{INTRODUCTION}

Abdominal tuberculosis (TB) is not uncommon in the developing world, but isolated colonic TB is rare; it has been estimated that $2-3 \%$ of patients with abdominal TB have isolated colonic involvement (1). It also accounts for $10.8 \%$ of all gastrointestinal TB with the commonest affected site being the transverse colon, followed by rectum and ascending colon (2). It commonly presents with vague abdominal pain, fever, weight loss, diarrhea and abdominal mass. In more than two thirds of affected patients, there is no evidence of pulmonary TB (으). We report a case of ascending colon tuberculosis masquerading as carcinoma.

\section{CASE REPORT}

A 40-year-old male presented with 6-month history of vague right-sided abdominal pain, diarrhoea, and weight loss. Clinical examination revealed cachexia, pallor but no lymphadenopathy or jaundice. He was apyrexial with a palpable mobile mass in the right hypochondrium which was non-tender and measured $7 \times 5 \mathrm{~cm}$. Blood tests revealed hypochromic microcytic anaemia. Tumor markers, renal and hepatic panels were normal. His chest x-ray was normal, but computed tomography (CT) scan of the abdomen revealed a large polypoid mass lesion in the right colon extending from the ileo-caecal valve with lumen-narrowing and mildly enlarged pericolic lymph nodes. The liver was grossly normal. Colonoscopy revealed a polypoidal mass in the proximal part of the ascending colon. Biopsies revealed inflammatory changes but no evidence of malignancy. Despite negative colonoscopic biopsies, a provisional diagnosis of right colonic cancer was made and he was prepared for right hemicolectomy. 


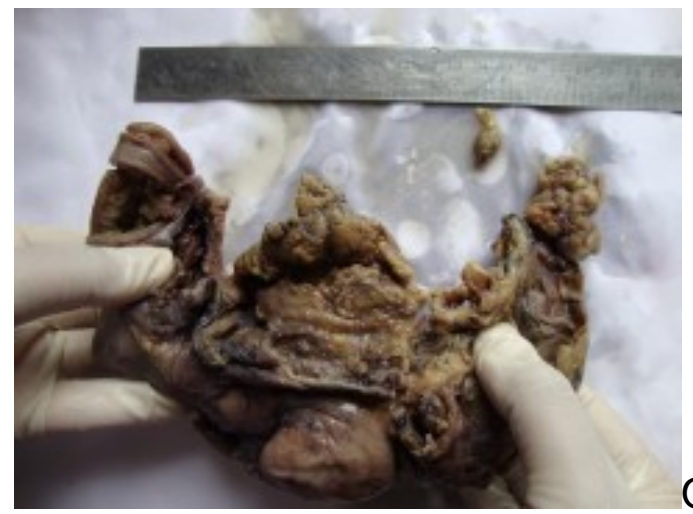

On laparotomy, the mass in the subhepatic area was recognized with minimal free straw-colour peritoneal fluid. A provisional diagnosis of carcinoma colon was made. Hence, a right hemicolectomy was performed with an ileo-transverse anastomosis, and the specimen was sent for gross examination and histopathology (Figure 1 and Figure 2). Histopathology revealed caseating granulomas diagnostic of TB, with no evidence of malignant cells. He was started on anti-tuberculous therapy consisting of daily doses of isoniazid $300 \mathrm{mg}$, rifampicin $600 \mathrm{mg}$ and ethambutol $900 \mathrm{mg}$ for two months, followed by four months of isoniazid and rifampicin therapy; the whole treatment lasted six months. The patient made a complete recovery without complications and is currently on regular follow-up.

\section{DISCUSSION}

Abdominal TB continues to be a major health problem in the developing world. The incidence of colon TB is increasing with the rise in numbers of high risk patients such as HIV-infected individuals, patients with chronic renal disease, and immunosuppressed patients on immunosuppressive or prolonged steroid therapy. Most reported colonic TB cases in non-immunosuppressed individuals come from the Indian subcontinent (1- $\underline{3})$. The most common presenting symptom and abnormal physical sign are abdominal pain and abdominal mass ( $90 \%$ and $58 \%$ of patients, respectively) (4). Varied and nonspecific presenting symptoms make early diagnosis difficult and elusive with subsequent delays in management. To avoid such a dangerous delay in management, some authors recommend a trial of anti-tuberculous chemotherapy in patients with high clinical suspicion on the basis of colonoscopic appearance alone, even in the absence of the classic features of TB, after ruling out other causes, especially cancer and Crohn's disease by histopathological examination $(1, \underline{3}$ ). TB and colon cancer may, however, coexist $(\underline{4}, \underline{5})$. This coexistence may be of high frequency necessitating the need for epidemiological and histopathological investigations into the aetiological relationship between the two diseases (ㅁ). Colonoscopic examination with combined histopathologic and bacteriologic study of biopsy specimens is a powerful tool for the diagnosis of intestinal TB in $60 \%$ of patients without the need for resectional surgery (1). However, the colonoscopic features are quite variable, but typically are those of circumferential ulceration or a single ulcer with an elevated or nodular margin surrounded by hyperemic mucosa (3). Other colonoscopic features include nodules, deformed segment of the colon, polypoid lesions or pseudopolyps, strictures, and less commonly, fibrous bands forming mucosal bridges (므). Like in this case, tuberculous lesions may mimic carcinoma in $20 \%$ of cases and acid-fast bacilli (AFB) may not be isolated at all (4). Nevertheless, polymerase chain reaction (PCR) analysis of a colonic biopsy may reveal AFB of the mycobacterium tuberculosis species. It is a rapid, sensitive, and specific method in the diagnosis of intestinal 
TB. It is also valuable in the differentiation between intestinal TB and Crohn's disease (7). Imaging plays a diagnostic role with features that suggest the correct diagnosis. Such features are cecal amputation, ileocecal thickening and inflammation, shortening of the ascending colon, gaping of the ileocecal valve, mesenteric adenopathy, a misty mesentery, diffuse omental infiltration, loculated high-density ascites, an enhancing peritoneum with or without an omental line, nodularity of the surface of the mesenteric leaves, and transperitoneal permeation (ㅇ) . Other commonly observed radiological findings include strictures (54\%), colitis $(39 \%)$ and less frequently polypoid lesions (7\%) (). . Complications noted were in the form of perforations and fistulae in less than $20 \%$ of cases (2). This case highlights the ease with which colonic TB may be mistaken for colonic cancer and the dilemma of diagnosing it radiologically and endoscopically. The clinical features of both these conditions are identical and, occasionally, only histopathology may provide the final diagnosis.

\section{REFERENCES}

1. Bhargava DK, Kushwaha AK, Dasarathy S, Shriniwas, Chopra P: Endoscopic diagnosis of segmental colonic tuberculosis. Gastrointest Endosc; 1992; 38: 571-4

2. Nagi B, Kochhar R, Bhasin DK, Singh K: Colorectal tuberculosis. Eur Radiol; 2003; 13(8): 1907-12

3. Alvares JF, Devarbhavi H, Makhija P, Rao S, Kottoor R: Clinical, colonoscopic, and histological profile of colonic tuberculosis in a tertiary hospital. Endoscopy; 2005; 37(4): $\underline{351-6}$

4. Kaushik R, Sharma R, Attri AK: Coexisting tuberculosis and carcinoma of the colon: a report of two cases and a review of the literature. Trop Gastroenterol; 2003; 24(3): 137-9

5. Leong AF, Seow-Choen F, Goh HS: Colorectal cancer and intestinal tuberculosis. Ann Acad Med Singapore: 1993; 22(6): 934-6

6. Jain BK, Chandra SS, Narasimhan R, Ananthakrishnan N, Mehta RB: Coexisting tuberculosis and carcinoma of the colon. Aust N Z J Surg: 1991; 61(11): 828-3

7. Gan H, Ouyang Q, Bu H, Li S, Chen D, Li G, et al.: Value of polymerase chain reaction assay in diagnosis of intestinal tuberculosis and differentiation from Crohn's disease. Chin Med J (Engl): 1995; 108(3): 215-20

8. Jadvar H, Mindelzun RE, Olcott EW, Levitt DB: Still the great mimicker: abdominal tuberculosis. AJR Am J Roentgenol; 1997; 168(6): 1455-60 